\title{
La contribución del turismo al crecimiento económico de la ciudad de Medellín - Colombia*
}

\author{
Recibido: 10 de abril de 2020 - Aceptado: 29 de agosto de 2020
}

Doi: https://doi.org/10.12804/revistas.urosario.edu.co/economia/a.8926

\author{
Juan Gabriel Brida ${ }^{+}$ \\ Martín Alberto Rodríguez Brindis ${ }^{\ddagger}$ \\ Maria Leivy Mejía-Alzate $\$$
}

\section{Resumen}

El objetivo de este trabajo es medir la contribución del turismo al crecimiento económico de la ciudad de Medellín para el periodo 2005-2015. Inicialmente, las diferentes ramas características del sector turístico se identifican en las cuentas locales del Producto Interno Bruto (РIB), luego se mide la contribución del turismo al crecimiento económico en Medellín y, se determina la relación entre las actividades turísticas y el crecimiento económico de la ciudad, utilizando la metodología introducida por Ivanov y Webster (2007). Los resultados muestran que para el periodo analizado, la participación del turismo en

* Este artículo presenta algunos resultados del proyecto "Medición del Impacto Económico y Social del Turismo en Medellín" financiado por la Subsecretaría de Turismo de la Alcaldía de Medellín, a través de la Federación Nacional de Comerciantes, FenAlco Antioquia y la Institución Universitaria Colegio Mayor de Antioquia.

† Universidad de la República, Uruguay. oRCiD: https://orcid.org/0000-0001-62681604

$\ddagger$ Universidad Anáhuac de Oaxaca, México. orcid: https://orcid.org/0000-00016268-1604

$\S$ Institución Universitaria Colegio Mayor de Antioquia, Medellín. Dirección: Cra 78 \# 65-46, Medellín, Colombia. Teléfono: +57 (4) 44456 11, ext: 147. Email: maria.mejia@ colmayor.edu.co oRCID: https://orcid.org/0000-0003-4631-8113

Para citar este artículo: Brida, J. G., Rodríguez Brindis, M., \& Mejía-Alzate, M. L. (2021). La contribución del turismo al crecimiento económico de la ciudad de Medellín - Colombia. Revista de Economía del Rosario, 24(1), 21-43. https://doi.org/10.12804/revistas.urosario. edu.co/economia/a.8926 
el PІв de Medellín estuvo entre el 8 y 9\%, lo que evidencia la importancia del sector para la economía de la ciudad.

Palabras clave: contribución del turismo al crecimiento económico; economía del turismo; impacto económico del turismo; turismo en Medellín.

Clasificación JEL: E01, F43, O54, R28, Z32.

\title{
The Contribution of Tourism to the Economic Growth of Medellin - Colombia
}

\begin{abstract}
This work aims to measure tourism's contribution to economic growth in the city of Medellin, Colombia, using data for the period 2005-2015. Initially, the different characteristic of the tourism sector branches are identified in the local GDP accounts. Then, tourism's contribution to economic growth in Medellin is measured, and the relationship between tourism activities and economic growth is determined using the methodology of Ivanov and Webster (2007). The results show that for the analyzed period, the participation of tourism in Medellin's GDP was between 8 and $9 \%$, which illustrates the importance of the sector for the economy of the city.
\end{abstract}

Keywords: Economic impact of tourism; tourism economics; tourism contribution to economic growth.

JEL classification: E01, F43, O54, R28, Z32.

\section{A contribuição do turismo para o crescimento econômico da cidade de Medellín-Colômbia}

Resumo

O objetivo deste trabalho é medir a contribuição do turismo para o crescimento econômico da cidade de Medellín-Colômbia no período 2005-2015. Inicialmente, os diferentes ramos característicos do setor turístico são identificados nas contas locais do Produto Interno Bruto (PIB), depois mede-se a contribuição do turismo para o crescimento econômico de Medellín e determina-se a relação entre as atividades turísticas e o crescimento econômico da cidade usando a metodologia apresentada em Ivanov e Webster (2007). Os resultados mostram que, para o período analisado, a participação do turismo no PIB de Medellín ficou entre 8 e $9 \%$, o que mostra a importância do setor para a economia da cidade.

Palavras-chave: economia do turismo; impacto econômico do turismo; contribuição do turismo para o crescimento econômico; turismo em Medellín.

Classificação JEL: E01, F43, O54, R28, Z32. 


\section{Introducción}

El turismo es reconocido como un sector que tiene un impacto positivo sobre el crecimiento y desarrollo económico ${ }^{1}$ a pesar del problema que genera su estacionalidad para las actividades de este sector (Cisneros-Martínez \& Fernández-Morales, 2016). Cuando la actividad turística crece, los visitantes aumentan y gastan más dinero en el destino, lo cual conduce a un incremento del PIB y al crecimiento económico del lugar (Webster \& Ivanov, 2014).

Los principales impactos económicos positivos del turismo están relacionados con los ingresos por divisas, la contribución a los ingresos públicos, la generación de empleo y las oportunidades de negocio (Brida et al., 2008). Según los mismos autores, Brida et al. (2008), existen tres tipos de impactos económicos del turismo: directos, indirectos e inducidos. El impacto directo es el generado por las ventas de las empresas de servicios turísticos. El impacto indirecto está relacionado con la compra y venta de bienes y servicios de proveedores directos de las empresas turísticas a otras empresas de la región, quienes continúan la cadena cuando compran insumos a otras empresas. Por último, los efectos inducidos, son los relacionados con el gasto realizado por los propietarios y empleados de las empresas - turísticas y no turísticas-, es decir, los efectos inducidos se presentan cuando los destinatarios del gasto directo e indirecto gastan sus ingresos.

Medir los efectos directos, indirectos e inducidos del turismo en el crecimiento económico es difícil, debido a que su contribución se encuentra repartida en diferentes sectores como el agrícola, manufactura, servicios financieros, entre otros (Brida et al., 2011; Brida et al., 2008; Brida et al., 2007; Brida, Monterubbianesi et al., 2010; Brida, Pereyra et al., 2010; CuadradoRoura \& López-Morales, 2011).

Existen dos contextos para medir la contribución del turismo en una economía: la contribución pasada (ex-post) y la contribución al crecimiento en el largo plazo (ex-ante). Los análisis de contribución pasada o ex-post, se realizan cuando el consumo turístico y otros elementos de la demanda turística han ocurrido, es decir, los cálculos se realizan con base en datos pasados. Por el contrario, la contribución ex-ante o contribución en el largo plazo, conocida también como la hipótesis del turismo como motor del crecimiento económico — tourism led-growth hypothesis, TLGH-, consiste en

1 Véase Balaguer y Cantavella-Jordá (2002), Bendodo Benasayag et al. (2019), Brida et al. (2017), Capó Parrilla et al. (2007), De La O Barroso González y Flores Ruiz (2006), Dritsakis (2004), Durbarry (2004), Ishikawa y Fukushige (2007), Kim et al. (2006), Meyer Krumholz (2004) y Mukherjee y Dutta (2018). 
proyectar la contribución futura del turismo con base en eventos o situaciones que posiblemente ocurran, tales como nuevas políticas públicas, construcción de nuevos escenarios deportivos y de diversión, nuevas campañas promocionales, entre otras. Sin embargo, en este trabajo no se hablará sobre la contribución en el largo plazo, el lector interesado puede remitirse a los trabajos de revisión de la literatura en la TLGH. ${ }^{2}$

Sobre la contribución pasada, existen tres técnicas básicas de análisis: modelo basado en el análisis insumo-producto (Input-Output Model), modelo de equilibrio general computable (Computable General Equilibrium, CGE) y la metodología de Ivanov y Webster.

El análisis insumo-producto se concentra en la industria y sus relaciones directas con otros sectores económicos. El modelo de equilibrio general computable tiene en cuenta un modelo de insumo-producto, pero la diferencia radica en que el CGE partiendo de un equilibrio general de la economía, modela los cambios en la contribución económica generados por cambios en las variables como precios, cambios en la demanda, cambios en la política, entre otros (Dwyer et al., 2004). Finalmente, la metodología de Ivanov y Webster, conocida como Metodología de Descomposición del Crecimiento, desagrega el crecimiento económico generado por el turismo y por otras industrias para calcular el incremento del PIB real per cápita, como medida del crecimiento económico de un país (Ivanov \& Webster, 2007). Es en esta línea en la que se enmarca el presente trabajo de investigación.

La Metodología de Descomposición del Crecimiento ha sido utilizada por diversos autores en varios países. Ivanov y Webster (2007) la aplicaron en España, Grecia y Chipre. Brida et al. (2007) utilizan la metodología para el caso mexicano y la comparan con el mismo indicador para Argentina, Brasil y Uruguay. Así mismo, Brida et al. (2008) aplican este método en Reino Unido, Italia, Francia, España y Estados Unidos. A su vez, Such-Devesa et al. (2009) la utilizan para Colombia comparando sus resultados con Argentina, Brasil, México y Uruguay. Por su parte, Ivanov y Webster (2010) emplean la metodología para analizar la contribución de industrias específicas al crecimiento económico de Bulgaria para el periodo 2000-2005, su propósito fue usar la metodología propuesta para el turismo y generalizarla para todas las industrias de una economía nacional, permitiendo las comparaciones interindustriales. Brida et al. (2011) aplican el método en las principales regiones turísticas de Colombia. Posteriormente, Ivanov y Webster (2013a) usan la metodología para 174 países entre el 2000 y el 2010, con el fin de medir el

2 Véase Brida et al. (2016), Castro-Nuño et al. (2013) y Pablo-Romero y Molina (2013). 
impacto del turismo en el crecimiento económico a escala global sobre una base de país por país. De igual manera, en Ivanov y Webster (2013b) miden el impacto de la globalización sobre la contribución del turismo al crecimiento económico con datos de 167 países. Del mismo modo, Llorca Rodríguez et al. (2013) implementan la metodología de Ivanov y Webster para determinar el rol del turismo en el crecimiento económico de la región de Arequipa, Perú.

Asimismo, Uribe y Gaitán Guerrero (2013) la emplean para determinar el efecto de la Inversión Extranjera Directa (IED) en el crecimiento económico del sector hotelero colombiano. En Webster e Ivanov (2014), se busca determinar el impacto de la competitividad de un destino sobre la contribución del turismo al crecimiento económico, para ello, miden la competitividad de un lugar con el índice de competitividad de viajes y turismo del Foro Económico Mundial y la contribución del turismo al crecimiento económico con la Metodología de Descomposición del Crecimiento. En tanto, Rodríguez Brindis (2014) realiza una expansión de la metodología de Ivanov y Webster para medir la contribución individual que cada una de las ramas características del sector turístico mexicano tiene sobre el crecimiento económico del país. Por último, Brida et al. (2017) expanden la metodología utilizando datos de la Cuenta Satélite de Turismo de Colombia, para medir los efectos de cada una de las ramas características del sector turístico sobre el crecimiento económico colombiano.

Los trabajos mencionados muestran que en general, en el conjunto de los países analizados, los que presentan mayor participación de las actividades turísticas en el Valor Agregado Bruto (VAB) son los que tienen el mayor dinamismo y contribución al crecimiento económico de las nombradas actividades. Esto puede sugerir que es tan importante para el crecimiento económico un sector turístico desarrollado, como una buena integración de este al conjunto de las demás actividades económicas. Los trabajos revisados evidencian que el mayor efecto de un incremento en el turismo es alterar la composición de la actividad económica, y, por tanto, los agentes necesitan adoptar una visión más amplia para sostener el desarrollo del turismo en los destinos. Todos los trabajos, los numerosos datos y estimaciones aportadas, certifican que el sector del turismo sigue siendo un soporte insustituible de la economía de países desarrollados y no desarrollados, y que su rol continuará siendo importante en los próximos años, tanto para la recuperación económica como para contribuir a mejorar la competitividad conjunta de la economía. En suma, las investigaciones anteriores reflejan que la contribución del turismo al crecimiento económico no es constante, sino que presenta fluctuaciones, aunque estas no son tan importantes como en otros sectores de la economía. 
Partiendo de la limitación de la metodología de Ivanov y Webster, que solo mide los efectos directos del turismo sobre el crecimiento del PIB per cápita, sin tener en cuenta los efectos indirectos e inducidos que pueden tener en la economía, el objetivo general de este trabajo es medir la contribución del turismo al crecimiento económico, mediante una expansión de la propuesta de Ivanov y Webster partiendo de los datos del PIв de Medellín para el periodo 2005-2015. Inicialmente se identifican las diferentes ramas características del sector turístico en las cuentas del PIв local, luego, se mide la contribución del turismo sobre el crecimiento económico en Medellín y se determina de manera más específica la relación existente entre las actividades turísticas y el crecimiento económico. Los resultados muestran que para el periodo analizado, la participación del turismo en el PIв de Medellín estuvo entre el 8 y $9 \%$.

El trabajo se ha organizado de la siguiente forma: la sección uno presenta un breve recuento de la evolución del sector turístico en Medellín, sus aspectos institucionales y principales indicadores turísticos. La sección dos expone los datos y la metodología. La sección tres discute los resultados, y la última sección, muestra las principales conclusiones y recomendaciones de políticas de desarrollo.

\section{Turismo en Medellín}

Medellín como una de las ciudades principales de Colombia, ha incorporado el turismo en su agenda y cada día esta actividad se hace más importante para ella. El turismo ha impactado a la sociedad medellinense en el desarrollo de su vocación principal alrededor del turismo de reuniones, inclinación que poco a poco se ha ido complementando con el impacto de la ciudad transformada urbanísticamente, que atrae a turistas nacionales y extranjeros a otros productos turísticos como son naturaleza, cultura y salud.

En el 2000 se construyó el primer Plan de Desarrollo Turístico para la ciudad de Medellín, en el cual se plantearon los lineamientos iniciales del turismo, especialmente el de negocios, llevando a la creación del Medellín Convention and Visitors Bureau, Plaza Mayor en el 2006 y el Sistema de Información Turística (SITUR) en el 2007. Para el 2008 se consolidó la creación del Clúster de Turismo, Negocios, Ferias y Convenciones, como una estrategia para fortalecer y encadenar a las empresas de turismo dedicadas a eventos y

3 Se tomó el periodo 2005-2015 porque a la fecha de realización del estudio, son los únicos datos oficiales disponibles con los que cuenta el Departamento Administrativo de Planeación de Medellín. 
negocios. En ese mismo año, se creó el Parque Arví, como una apuesta de la ciudad en la línea de turismo de naturaleza (Alcaldía de Medellín \& Centro de Ciencia y Tecnología de Antioquia -CTA-, 2015).

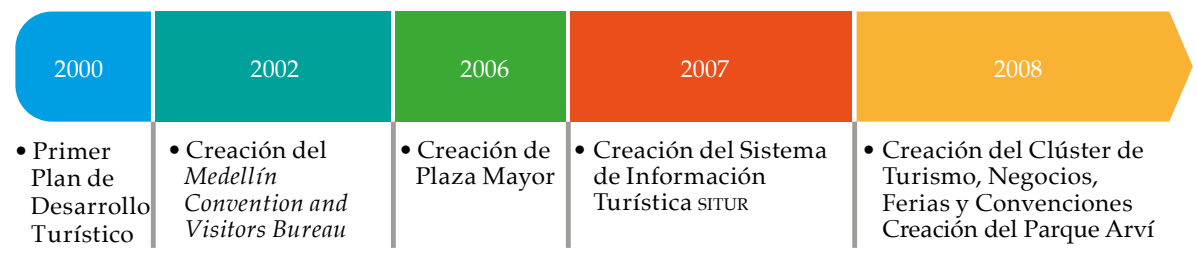

\begin{tabular}{|c|c|c|c|c|}
\hline 2011 & 2012 & 2013 & 2015 & 2017 \\
\hline $\begin{array}{l}\text { - Plan de } \\
\text { Desarrollo } \\
\text { Turístico Medellín } \\
\text { 2011-2016 } \\
\text { - Creación de la } \\
\text { mesa de } \\
\text { competitividad }\end{array}$ & $\begin{array}{l}\text { - Creación de la } \\
\text { Subsecretaría de } \\
\text { Turismo de la } \\
\text { ciudad de } \\
\text { Medellín }\end{array}$ & $\begin{array}{l}\text { - Política } \\
\text { grandes } \\
\text { eventos y } \\
\text { eventos de } \\
\text { conocimien- } \\
\text { tos }\end{array}$ & $\begin{array}{l}\text { - Política } \\
\text { Pública } \\
\text { de } \\
\text { Turismo }\end{array}$ & $\begin{array}{l}\text { - Creación, discusión y } \\
\text { actualización de: } \\
\text { Política Pública de } \\
\text { Desarrollo Económico, } \\
\text { Política Pública de Turismo } \\
\text { y Plan Estratégico de } \\
\text { Turismo de Medellín } \\
\text { 2018-2024 }\end{array}$ \\
\hline
\end{tabular}

Figura 1. Aspectos institucionales del turismo en Medellín Fuente: Mejía-Alzate et al. (2019).

En el 2011 se consolidó el Plan de Desarrollo Turístico 2011-2016 y nació la Mesa de Competitividad, como escenario para la gobernanza turística de la ciudad. Para el 2012, se creó la Subsecretaría de Turismo de la ciudad de Medellín como dependencia de la Secretaría de Desarrollo Económico. En el 2013 se aprobó la Política de turismo de grandes eventos y eventos de conocimiento para Medellín, y, en septiembre del 2015 nació la Política Pública de Turismo de la ciudad, la cual recoge todos los decretos relacionados con el turismo hasta ese momento.

Finalmente, en el 2017 se crea, discute y actualizan los principales lineamientos del sector turístico de la ciudad de Medellín: Política Pública de Desarrollo Económico, Política Pública de Turismo y Plan Estratégico de Turismo de Medellín 2018-2024.

La Política Pública de Desarrollo Económico del Municipio de Medellín, evidencia la importancia de la actividad turística para el desarrollo económico de la ciudad, identificándolo como un sector con amplio número de Mipymes. Así mismo, se refieren a él como un sector turístico transversal a las áreas de especialización definidas para la ciudad (Mejía-Alzate, 2019).

En ese sentido, la Política Pública de Turismo del Municipio de Medellín, identifica al turismo como una estrategia de desarrollo socioeconómico para la ciudad, donde la articulación público-privada es relevante para la 
planificación, gestión y posicionamiento de Medellín como un destino turístico sostenible (Mejía-Alzate, 2017).

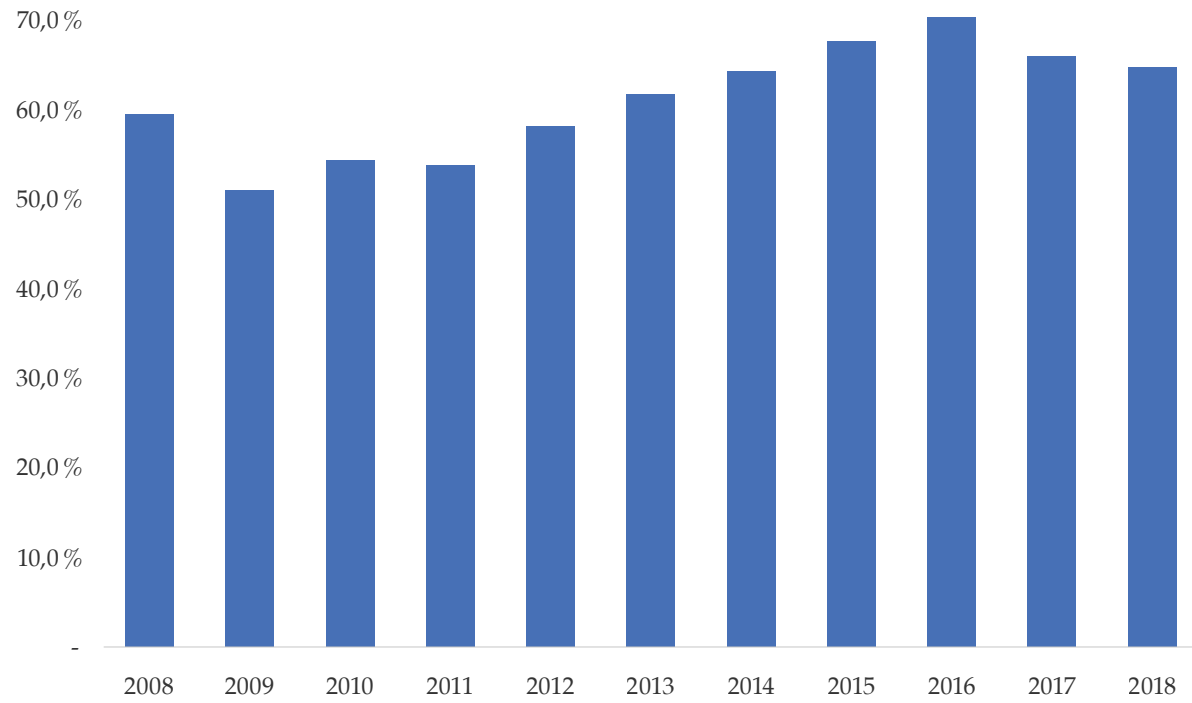

Figura 2. Porcentaje de ocupación hotelera en la ciudad de Medellín 2008-2018 Fuente: Fenalco Antioquia Operador del situr (2019).

Tabla 1. Evolución ingreso de visitantes nacionales y extranjeros por punto de Control Migratorio Regional José María Córdova 2008-2018

\begin{tabular}{cccc}
\hline Año & $\begin{array}{c}\text { Ingreso anual de } \\
\text { colombianos }\end{array}$ & $\begin{array}{c}\text { Ingreso anual de } \\
\text { extranjeros }\end{array}$ & $\begin{array}{c}\text { Total ingreso anual por } \\
\text { punto migratorio }\end{array}$ \\
\hline 2008 & 194.319 & 75.761 & 270.080 \\
2009 & 219.443 & 108.809 & 328.252 \\
2010 & 238.025 & 134.390 & 372.415 \\
2011 & 259.319 & 121.779 & 381.098 \\
2012 & 282.587 & 121.338 & 403.925 \\
2013 & 338.228 & 151.710 & 489.938 \\
2014 & 391.030 & 158.425 & 549.455 \\
2015 & 396.250 & 212.275 & 608.525 \\
2016 & 438.848 & 263.238 & 702.086 \\
2017 & 460.877 & 274.693 & 735.570 \\
2018 & 500.256 & 323.014 & 823.270 \\
\hline
\end{tabular}

Fuente: Fenalco Antioquia Operador del situr (2019). 
Los principales indicadores de ciudad para el periodo 2008-2018, como son el porcentaje de ocupación hotelera y el ingreso de visitantes nacionales y extranjeros, tuvieron una positiva evolución. El porcentaje de ocupación hotelera se caracteriza por estar encima del 55.3 \%, a excepción del periodo 2009-2012; la ciudad pasó de tener 270.080 visitantes nacionales y extranjeros en el 2008, a cerrar el 2018 con 823.270 visitantes. Estos indicadores han incentivado la inversión en diversos tipos de alojamientos, incremento de oferta que no se reflejó en variaciones negativas sobre el porcentaje de ocupación hotelera de la ciudad.

Según el Sistema de Indicadores Turísticos de Medellín - Antioquia (SITUR), el sector turístico de la ciudad de Medellín al 31 de agosto del 2018 contaba con 1.735 prestadores de servicios turísticos clasificados de la siguiente manera:

Tabla 2. Prestadores de servicios turísticos en la ciudad de Medellín

\begin{tabular}{lc}
\hline \multicolumn{1}{c}{ Tipo de prestador } & Cantidad \\
\hline Establecimientos de alojamiento y hospedaje & 790 \\
Agencia de viajes & 581 \\
Guía de turismo & 119 \\
Operadores profesionales de congresos, ferias y convenciones & 83 \\
Oficina de representación turística & 46 \\
Empresa de transporte terrestre automotor & 44 \\
Establecimiento de gastronomía y similares & 36 \\
Arrendadores de vehículos para turismo nacional e internacional & 30 \\
Empresas captadoras de ahorro para viajes y de servicios turísticos & 5 \\
Empresa de tiempo compartido y multipropiedad & 1 \\
Total general & 1.735 \\
\hline
\end{tabular}

Nota: Información con datos del Registro Nacional de Turismo a Agosto 31 del 2018. Fuente: SITUR (2018).

Los aspectos institucionales del turismo en Medellín muestran que desde el 2000 se comenzó a darle importancia al sector turístico, como una fuente dinamizadora del desarrollo socioeconómico de la ciudad, pero solo 11 años después se publicaron decretos relacionados con ello, los cuales son recogidos en la Política Pública de Turismo del 2015. Más adelante, en el 2017, en la Política Pública de Desarrollo Económico se evidencia la importancia de la actividad turística para la ciudad (Mejía-Alzate, 2019). Así mismo, la 
positiva evolución de los principales indicadores de la ciudad, refleja la incidencia de esos aspectos institucionales en el sector. Con el fin de monitorear el avance de las políticas públicas enfocadas en la promoción del sector turístico de la ciudad de Medellín, este trabajo busca medir la contribución del turismo al crecimiento económico de la ciudad con los datos suministrados por el Departamento Administrativo de Planeación de Medellín para el periodo 2005-2015.

\section{Metodología}

Para medir la contribución que el turismo tiene sobre el crecimiento económico, como se ha mencionado, se utiliza la metodología propuesta por Ivanov y Webster (2007), la ventaja de esta metodología es que la Descomposición del Crecimiento Económico genera una medida del desempeño de cada una de las ramas características del turismo, mostrando su efectividad individual en estimular el crecimiento real per cápita del PIB. Además, esta metodología permite la comparación entre las ramas características del turismo presentadas por las CST, sin tener que recurrir a modelos complejos de equilibrio general computable o modelos de input-output multisectoriales.

Por otro lado, cuando se limita únicamente a medir la participación del turismo en el PIB, podría darse el caso de que un aumento de la participación del turismo en la economía de un país o de una región, pueda ser el resultado del estancamiento de otras industrias, su reemplazo o expulsión por el turismo. En este sentido, la metodología utilizada hace posible medir el estímulo que el turismo genera en la tasa de crecimiento económico, y no solo la parte del PIB generada por el turismo. Lo que acerca a una medida más precisa del impacto del turismo en la economía.

Dicho lo anterior, un impacto se considera un beneficio o costo económico si aumenta el bienestar de la población local. Por lo tanto, el crecimiento económico debería aumentar el bienestar de la población local, aunque ciertos segmentos se beneficiarán más que otros. Por esto, la metodología propuesta por Ivanov y Webster (2007) utiliza el crecimiento del PIB real per cápita como medida del crecimiento económico:

$$
g_{t}=\left[\left(\frac{\frac{Y_{t\left(P_{b}\right)}}{N_{t}}}{Y_{t-1\left(P_{b}\right)}}\right)-1\right]
$$


Donde $Y_{t\left(P_{b}\right)}$ es el PIB en el periodo $t$ a precios constantes; $Y_{t-1\left(P_{b}\right)}$ es el PIB a precios constantes en el periodo $t-1 ; N_{t}$ y $N_{t-1}$ son el tamaño promedio de la población en el periodo $t$ y en el periodo $t-1$ respectivamente; y el subíndice $P_{b}$ representa el año base.

Si se desagrega la ecuación para separar el PIB turístico a precios constantes en el periodo $t\left(Y_{t\left(P_{b}\right)}^{T}\right)$ del PIB de otras industrias a precios constantes para el mismo periodo $\left(\sum_{R \neq T} Y_{t\left(P_{b}\right)}^{R}\right)$ y, el PIB turístico a precios constantes en el periodo $t-1\left(Y_{t-1\left(P_{b}\right)}^{T}\right)$ del PIB de otras industrias a precios constantes en el mismo periodo $\left(\sum_{R \neq T} Y_{t-1\left(P_{b}\right)}^{R}\right)$, se tendrá que la tasa de crecimiento del PIB real per cápita en el tiempo $t$ es:

$$
g_{t}=\left[\frac{\frac{Y_{t\left(P_{b}\right)}^{T}}{N_{t}}-\frac{Y_{t-1\left(P_{b}\right)}^{T}}{N_{t-1}}}{\frac{Y_{t-1\left(P_{b}\right)}}{N_{t-1}}}+\frac{\frac{\sum_{R \neq T} Y_{t\left(P_{b}\right)}^{R}}{N_{t}}-\frac{\sum_{R \neq T} Y_{t-1\left(P_{b}\right)}^{R}}{N_{t-1}}}{\frac{Y_{t-1\left(P_{b}\right)}}{N_{t-1}}}\right]
$$

Con el fin de medir la contribución individual que cada una de las actividades económicas tiene sobre el crecimiento, Rodríguez Brindis (2014) propone una expansión de la ecuación (2) que al ser desarrollada se obtiene:

$$
g_{t}^{T}=\frac{\frac{\sum_{R C} Y_{t\left(P_{b}\right)}^{R C}}{N_{t}}-\frac{\sum_{R C} Y_{t-1\left(P_{b}\right)}^{R C}}{N_{t-1}}}{\frac{Y_{t-1\left(P_{b}\right)}}{N_{t-1}}}
$$

Donde $Y_{t\left(P_{b}\right)}^{R C}$ y $Y_{t-1\left(P_{b}\right)}^{R C}$ representan la producción bruta de alguna de las ramas características ya mencionadas.

Es importante resaltar que este método solo permite medir los efectos directos que cada una de las ramas características del turismo tienen sobre el рів real per cápita, dejando de lado los posibles impactos indirectos y los efectos inducidos que estos pueden tener sobre la economía real. Lo anterior es una limitación de esta metodología, en cuanto a la estimación de los impactos del turismo sobre el crecimiento económico. Como señalan Brida et al. (2008): "El aporte original del método es brindar una estimación ex post (lo que realmente sucedió) de la contribución del turismo sobre el crecimiento del PIB" (p. 37). 
Una particularidad del método es que, una vez los datos estadísticos necesarios son obtenidos, puede ser aplicado para otras industrias y con ello, permitir un comparativo de la contribución que cada una aporta al crecimiento económico.

Otra bondad de este método es que a través de la ecuación (3) se puede desagregar cualquier sector industrial, tanto como la disponibilidad de datos lo permita. En este aspecto, el presente trabajo desagrega al sector turístico en sus ramas características siguiendo las recomendaciones internacionales para estadísticas de turismo de la Organización Mundial del Turismo (омт, 2015), que definen las principales actividades de la industria turística. ${ }^{4}$ Identificando estas ramas, la suma de su Valor Agregado (va) es una variable representativa del turismo, más robusta que el utilizar únicamente información de hoteles y restaurantes. Las ramas características del turismo identificadas para Medellín son presentadas en la tabla 3:

Tabla 3. Ramas características del turismo acorde a clasificación de la омт

34. Hoteles, restaurantes, bares y similares

35. Transporte por vía terrestre

37. Transporte por vía aérea

38. Actividades complementarias y auxiliares al transporte; actividades de agencias de viajes 46. Actividades de asociaciones no clasificado previamente (n.c.p); actividades de esparcimiento y actividades culturales y deportivas; otras actividades de servicios de mercado

Fuente: elaboración propia de acuerdo con los datos suministrados por el Departamento Administrativo de Planeación de Medellín (2019).

Adicionalmente, buscando comparar el aporte del turismo al crecimiento económico de la ciudad con otras actividades económicas, se identificaron y tomaron datos de los siguientes sectores en las cuentas del PIB para Medellín:

Tabla 4. Otros sectores de la economía
A. Agricultura, ganadería, silvicultura y pesca
B. Explotación de minas y canteras
C. Industrias manufactureras
D. Suministro de electricidad, gas y agua

Continúa

4 Las actividades de la industria turística son aquellas que constituyen un alto porcentaje del gasto del viajero - el alojamiento-, o de los ingresos del oferente de bienes y servicios a los turistas - artesanías y servicios de aeropuertos-. 


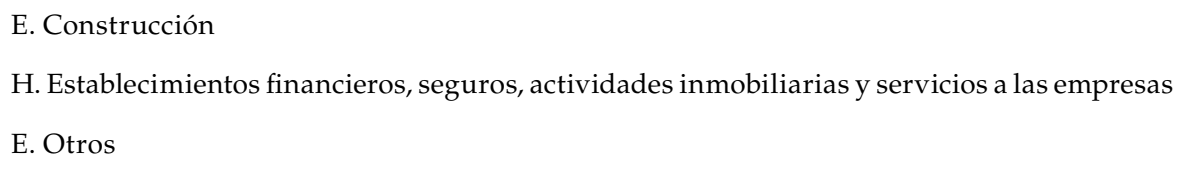

Fuente: elaboración propia de acuerdo con los datos suministrados por el Departamento Administrativo de Planeación de Medellín (2019).

\section{Resultados}

Para calcular la contribución del turismo al crecimiento económico utilizando la ecuación (3), se tomaron los datos del PIB y de la población para Medellín, suministrados por el Departamento Administrativo de Planeación de Medellín. Es de resaltar que las variaciones del PIB de Medellín 2005-2015, fueron realizados por la Unión Temporal Centanaro-Mahecha y el Departamento Administrativo de Planeación del Municipio de Medellín, conservando toda la metodología del Departamento Administrativo Nacional de Estadísticas de Colombia (DANE) para calcular las cuentas nacionales.

Los resultados de la aplicación de la metodología se presentan en la tabla 5, en la que se indica para cada año el crecimiento real del PIB de Medellín, medido por el Valor Agregado (vA) de la actividad y la contribución económica que los diferentes sectores aportan a dicho crecimiento.

Tabla 5. Contribución del turismo y otros sectores de la economía al crecimiento económico real per cápita de Medellín 2006-2015

\begin{tabular}{|c|c|c|c|c|c|c|c|c|c|c|}
\hline Periodo & 2006 & 2007 & 2008 & 2009 & 2010 & 2011 & 2012 & 2013 & 2014 & 2015 \\
\hline $\begin{array}{l}\text { Crecimiento } \\
\text { del va de } \\
\text { Medellín }\end{array}$ & $6.649 \%$ & $6.217 \%$ & $3.006 \%$ & $2.270 \%$ & $3.976 \%$ & $6.749 \%$ & $3.231 \%$ & $3.275 \%$ & o $3.507 \%$ & $2.989 \%$ \\
\hline Turismo & $0.481 \%$ & $0.772 \%$ & $0.554 \%$ & $0.047 \%$ & $0.142 \%$ & $0.162 \%$ & $0.331 \%$ & $0.173 \%$ & $0.222 \%$ & $0.388 \%$ \\
\hline Agricultura & $0.069 \%$ & $0.063 \%$ & $0.048 \%$ & $-0.010 \%$ & $0.007 \%$ & $0.087 \%$ & $0.055 \%$ & $-0.011 \%$ & $0.027 \%$ & $0.118 \%$ \\
\hline Minería & $0.006 \%$ & $0.005 \%$ & $-0.003 \%$ & $-0.003 \%$ & $0.000 \%$ & $0.005 \%$ & $-0.009 \%$ & $0.006 \%$ & $0.000 \%$ & $0.002 \%$ \\
\hline Manufactura & $1.270 \%$ & $0.721 \%$ & $-0.076 \%$ & $1.064 \%$ & $0.922 \%$ & $1.390 \%$ & $-0.813 \%$ & o $0.924 \%$ & $0.700 \%$ & $0.420 \%$ \\
\hline $\begin{array}{l}\text { Suministro de } \\
\text { electricidad, } \\
\text { gas y agua }\end{array}$ & $0.027 \%$ & $0.166 \%$ & $-0.013 \%$ & $0.014 \%$ & $-0.018 \%$ & $0.094 \%$ & $0.014 \%$ & $-0.019 \%$ & o $-0.080 \%$ & $-0.071 \%$ \\
\hline Construcción & $0.519 \%$ & $0.839 \%$ & $-1.026 \%$ & $-0.226^{\circ}$ & $-0.182^{\circ}$ & $0.701 \%$ & $-0.855 \%$ & $0.638 \%$ & $-0.302 \%$ & 0.239 \\
\hline
\end{tabular}




\begin{tabular}{lllllllllll}
\hline \multicolumn{1}{c}{ Periodo } & 2006 & 2007 & 2008 & 2009 & 2010 & 2011 & 2012 & 2013 & 2014 & 2015 \\
\hline $\begin{array}{l}\text { Estableci- } \\
\text { mientos } \\
\text { financieros, } \\
\text { seguros, } \\
\text { actividades } \\
\text { inmobiliarias } \\
\text { y servicios a } \\
\text { las empresas }\end{array}$ & $1.972 \%$ & $1.876 \%$ & $1.796 \%$ & $0.484 \%$ & $1.462 \%$ & $2.092 \%$ & $2.835 \%$ & $0.604 \%$ & $1.640 \%$ & $1.421 \%$ \\
Otros & & & & & & & & & & \\
\end{tabular}

Fuente: elaboración propia de acuerdo a los datos suministrados por el Departamento Administrativo de Planeación de Medellín (2019).

Por tanto, la tabla 5 muestra la desagregación en diversas industrias, incluyendo el turismo, respecto al crecimiento real económico de Medellín, por lo que dicha aportación por industria puede ser llamada como su contribución al crecimiento real económico. En consecuencia, los datos de la tabla 5 deben ser interpretados de la siguiente manera: tomando como ejemplo el 2015, Medellín tuvo un crecimiento real del PIв per cápita del $2.989 \%$ —columna 2015, renglón crecimiento del VA de Medellín-, de los cuales 0.388 \% — columna 2015, renglón Turismo-, pueden ser vinculados directamente al crecimiento de las actividades turísticas. Para este mismo año, si se toma como ejemplo el sector Construcción se tiene que $0.239 \%$ de los $2.989 \%$ del crecimiento del PIв real per cápita de la ciudad, son vinculados directamente con esta actividad — columna 2015, renglón Construcción- Nótese que la suma de las contribuciones de todas industrias da el total del crecimiento económico de Medellín.

Con el fin de facilitar la interpretación de los resultados, estos pueden ser expresados como la contribución porcentual que cada sector tiene sobre el 100 \% del crecimiento económico real de Medellín. Dichos porcentajes son presentados en la tabla 6. Para la obtención de estos resultados, se tomó la información presentada en la tabla 5 realizando una regla de tres. Por ejemplo, se tomó nuevamente el dato de la contribución al crecimiento económico del sector Turismo para el 2015 (0.388\%) y el dato del crecimiento económico real para Medellín en el mismo año (2.989\%), la contribución porcentual de este sector se calcularía de la siguiente manera: para el sector turístico $0.388 / 2.989 * 100$, obteniendo con ello un $13 \%$, que puede ser interpretado como que el sector turístico contribuye al $13 \%$ del total de crecimiento real económico de Medellín en el 2015. De este modo, la tabla 6 permite de forma sencilla comparar la contribución que los diferentes sectores de la economía tienen sobre el crecimiento económico de Medellín. 
Tabla 6. Contribución porcentual del turismo y otros sectores de la economía al crecimiento económico real per cápita de Medellín 2006-2015

\begin{tabular}{|c|c|c|c|c|c|c|c|c|c|c|}
\hline Periodo & 2006 & 2007 & 2008 & 2009 & 2010 & 2011 & 2012 & 2013 & 2014 & 2015 \\
\hline $\begin{array}{l}\text { Crecimien- } \\
\text { to del va de } \\
\text { Medellín }\end{array}$ & $100 \%$ & $100 \%$ & $100 \%$ & $100 \%$ & $100 \%$ & $100 \%$ & $100 \%$ & $100 \%$ & $100 \%$ & $100 \%$ \\
\hline Turismo & $7.24 \%$ & $12.42 \%$ & $18.43 \%$ & $2.08 \%$ & $3.58 \%$ & $2.41 \%$ & $10.25 \%$ & $5.29 \%$ & $6.34 \%$ & $13.00 \%$ \\
\hline Agricultura & $1.04 \%$ & $1.01 \%$ & $1.59 \%$ & $-0.44 \%$ & $0.18 \%$ & $1.29 \%$ & $1.71 \%$ & $-0.32 \%$ & $0.78 \%$ & $3.96 \%$ \\
\hline Minería & $0.10 \%$ & $0.08 \%$ & $-0.09 \%$ & $-0.14 \%$ & $0.01 \%$ & $0.07 \%$ & $-0.29 \%$ & $0.19 \%$ & $0.00 \%$ & $0.07 \%$ \\
\hline Manufactura & $19.11 \%$ & $11.60 \%$ & $-2.52 \%$ & $46.86 \%$ & $23.19 \%$ & $20.59 \%$ & $-25.17 \%$ & $28.21 \%$ & $19.95 \%$ & $14.05 \%$ \\
\hline $\begin{array}{l}\text { Suministro } \\
\text { de electrici- } \\
\text { dad, gas } \\
\text { y agua }\end{array}$ & $0.41 \%$ & $2.67 \%$ & $-0.43 \%$ & $0.62 \%$ & $-0.46 \%$ & $1.40 \%$ & $0.43 \%$ & $-0.59 \%$ & $-2.29 \%$ & $-2.38 \%$ \\
\hline $\begin{array}{l}\text { Construc- } \\
\text { ción }\end{array}$ & $7.80 \%$ & $13.50 \%$ & $-34.13 \%$ & $-9.95 \%$ & $-4.58 \%$ & $10.39 \%$ & $-26.47 \%$ & $19.47 \%$ & $-8.60 \%$ & $7.98 \%$ \\
\hline $\begin{array}{l}\text { Estableci- } \\
\text { mientos } \\
\text { financieros, } \\
\text { seguros, } \\
\text { actividades } \\
\text { inmobilia- } \\
\text { rias y ser- } \\
\text { vicios a las } \\
\text { empresas }\end{array}$ & $29.66 \%$ & $30.17 \%$ & $59.74 \%$ & $21.31 \%$ & $36.77 \%$ & $31.00 \%$ & $87.75 \%$ & $18.43 \%$ & $46.76 \%$ & $47.54 \%$ \\
\hline Otros & $34.64 \%$ & $28.54 \%$ & $57.41 \%$ & $39.65 \%$ & $41.32 \%$ & $32.86 \%$ & $51.80 \%$ & $29.33 \%$ & $37.07 \%$ & $15.78 \%$ \\
\hline
\end{tabular}

Fuente: elaboración propia a partir de los datos de la tabla 5.

De acuerdo a los resultados de la tabla se puede observar que en general, ha habido una tendencia al alza en la contribución del turismo al crecimiento económico durante este periodo, pasando de contribuir en un $7.24 \%$ en el 2006 a un $13 \%$ en el 2015, mostrando periodos con mayor porcentaje como el 2008 cuya contribución fue del 18.43 \%. Por otro lado, con las estimaciones obtenidas se puede considerar a la actividad turística en Medellín como un sector anticíclico, ya que en los años en que la contribución al crecimiento económico de la manufactura y construcción es negativa, el turismo contribuye de manera positiva, mitigando con ello la caída en el crecimiento real de la economía de Medellín.

La contribución del turismo al crecimiento económico de Medellín, no fue ajena al comportamiento del sector turístico nacional y al desempeño de la economía colombiana en general. Su comportamiento se podría dividir 
en tres periodos establecidos por años, el primero serían 2006, 2007 y 2008, cuya contribución fue $7.24 \%, 12.42 \%$ y $18.43 \%$, respectivamente. El segundo periodo entre el 2009, 2010 y 2011 cuya contribución fue de $2.08 \%, 3.58 \%$ y $2.41 \%$; y el tercero, el 2012, 2013, 2014 y 2015 con unos porcentajes de $10.25 \%$, $5.29 \%, 6.34 \%$ y $13 \%$.

En el primer periodo analizado, años 2006-2008, el sector turístico colombiano pasaba por una buena época, existían esfuerzos públicos y privados para generar espacios de desarrollo de iniciativas turísticas y, el turismo receptivo empezaba a mostrar un importante crecimiento del número de visitantes internacionales (Zapata-Aguirre et al., 2020); la ciudad de Medellín no fue ajena a esa tendencia nacional.

Finalizando el 2008 se presentó una fuerte caída del pIB colombiano, tal desempeño se vio acompañado del alejamiento de las metas de inflación, la caída en los sectores productivos tradicionales, el deterioro del mercado laboral, además del complejo panorama internacional y la posterior crisis económica mundial (Banco de la República de Colombia, 2009). En el 2009 la contribución del turismo al crecimiento económico de la ciudad de Medellín cayó fuertemente a $2.08 \%$, se mantuvo en $3.58 \%$ para el 2010 y $2.41 \%$ en el 2011; es probable que eso se explique en los impactos negativos de la crisis externa en la economía colombiana para el mismo periodo.

Para el periodo 2012-2015, las cifras del sector turístico nacional presentaban importantes crecimientos: la producción del sector de hoteles y restaurantes, las llegadas de turistas extranjeros, los ingresos de la cuenta de viajes de la balanza de pagos, la inversión extranjera directa en la industria turística y, el dinamismo que registra la construcción de hoteles llevando a que el pIB turístico fuera uno de los de mayor crecimiento, incluso por encima del рів nacional después de minas, construcción, servicios financieros y transporte (Díaz Olariaga, 2015). El desempeño del sector turístico no fue ajeno a esa realidad nacional, en el 2012, la contribución del turismo al crecimiento económico de la ciudad de Medellín aumentó a 10.25\% y llegó al $13 \%$ en el 2015.

Algo importante a considerar de la medición realizada en este trabajo, es la definición que se ha hecho de la variable turismo, con frecuencia este sector es medido utilizando la proxy de los subramas de actividad económica de hoteles y restaurantes, los cuales son ramas características del turismo; esto se debe principalmente a dos factores: primero, a la falta de información en muchos países sobre la totalidad del sector turístico; segundo, por lo complejo de la desagregación del sector en las metodologías utilizadas para evaluar el impacto del turismo en la economía de una región. 
Como se mencionó en la sección de metodología, el procedimiento utilizado en este trabajo tiene la bondad de poder desagregar el sector turístico en varias de sus ramas características, y no solo en hoteles y restaurantes (véase tabla 3). En tal sentido, la tabla 7 presenta los resultados de la contribución porcentual del turismo al crecimiento económico de Medellín, desde la definición de este trabajo y, la contrasta con la contribución porcentual al crecimiento económico de la rama de hoteles y restaurantes. Además, también se presenta la participación del turismo en el PIB de Medellín, el cual es un indicador frecuentemente utilizado para mostrar la relevancia del turismo en la economía.

Tabla 7. Comparativo de resultados para la ciudad de Medellín

\begin{tabular}{|c|c|c|c|c|c|c|c|c|c|c|}
\hline Periodo & 2006 & 2007 & 2008 & 2009 & 2010 & 2011 & 2012 & 2013 & 2014 & 2015 \\
\hline $\begin{array}{l}\text { Participación } \\
\text { porcentual va } \\
\text { del turismo en } \\
\text { el PIB de Mede- } \\
\text { llín }\end{array}$ & $8.48 \%$ & $8.71 \%$ & $9.00 \%$ & $8.84 \%$ & $8.64 \%$ & $8.25 \%$ & $8.31 \%$ & $8.21 \%$ & $8.15 \%$ & $8.29 \%$ \\
\hline $\begin{array}{l}\text { Participación } \\
\text { porcentual de } \\
\text { la rama hoteles } \\
\text { y restaurantes }\end{array}$ & $3.00 \%$ & $2.97 \%$ & $3.01 \%$ & $3.05 \%$ & $3.02 \%$ & $2.94 \%$ & $3.01 \%$ & $3.07 \%$ & $3.13 \%$ & $3.21 \%$ \\
\hline $\begin{array}{l}\text { Contribución del } \\
\text { turismo al creci- } \\
\text { miento económi- } \\
\text { co de Medellín }\end{array}$ & $7.24 \%$ & $12.42 \%$ & $18.43 \%$ & $2.08 \%$ & $3.58 \%$ & $2.41 \%$ & $10.25 \%$ & $5.29 \%$ & $6.34 \%$ & $13.00 \%$ \\
\hline $\begin{array}{l}\text { Contribución de } \\
\text { hoteles y restau- } \\
\text { rantes al creci- } \\
\text { miento económi- } \\
\text { co de Medellín }\end{array}$ & $2.06 \%$ & $2.42 \%$ & $4.45 \%$ & $4.47 \%$ & $2.27 \%$ & $1.83 \%$ & $5.03 \%$ & $4.97 \%$ & $4.93 \%$ & $5.77 \%$ \\
\hline
\end{tabular}

Fuente: elaboración propia de acuerdo con los datos suministrados por el Departamento Administrativo de Planeación de Medellín (2019).

La tabla 7 confirma que el turismo siempre aporta positivamente al crecimiento económico de Medellín y, que la rama de hoteles y restaurantes sigue este mismo comportamiento. De la misma forma, el turismo, visto como el total del valor agregado de sus ramas características, si bien contribuye positivamente al crecimiento económico, muestra mayor variabilidad en dicha contribución, mientras que la rama hoteles y restaurantes muestra una aportación prácticamente constante durante el periodo de análisis, lo que demuestra que es una rama robusta y consolidada dentro de la economía de Medellín. No obstante, las estimaciones ponen en evidencia que el 
tomar únicamente la rama de hoteles y restaurantes como una variable proxy de la actividad turística, subestima los efectos directos del turismo en la economía de Medellín.

\section{Conclusiones y recomendaciones de políticas de desarrollo}

Analizar la contribución del turismo al crecimiento económico de Medellín es significativo porque evidencia la importancia del sector para la ciudad, así como justifica la inversión pública y privada en esta industria. El crecimiento del turismo en Medellín ha acompañado el crecimiento de toda la economía, pues nunca hay aportes negativos como sí se ve en la mayoría de otros sectores, manifestando la regularidad del turismo como un factor de crecimiento, lo que corrobora la inversión en turismo como un espacio de administración financiera de riesgo.

En cuanto al porcentaje del crecimiento debido al turismo, se ve que hay movimientos irregulares en el 2009-2010-2011. Durante esos años se observa que el turismo contribuyó menos al crecimiento de la economía, la cual tuvo un desempeño peor que los años anteriores. Es de resaltar que durante ese tiempo hubo una contribución muy importante al crecimiento económico de la ciudad por parte de la industria manufacturera. Además, es de destacar, que el turismo siempre aporta positivamente al crecimiento económico de la ciudad con un promedio de más del $8 \%$ en el periodo analizado; se destacan el 2007, 2008, 2012 y 2015. Los resultados parecen mostrar las buenas prácticas de las políticas de desarrollo del sector en la ciudad, y por tanto, sería importante mantenerlas e incentivarlas. Como se ve en otros trabajos, se podría inferir que la contribución del turismo al crecimiento y participación en la economía debería ser mayor a los resultados obtenidos, ya que solo fueron medidos los efectos directos dejando a un lado los indirectos.

Las políticas turísticas de desarrollo regional deben contemplar en términos generales la subvención al desarrollo, la accesibilidad crediticia, el mejoramiento del sistema de transporte, el desarrollo de tecnologías, el desarrollo estadístico, el ordenamiento territorial y el mejoramiento de la seguridad. Desde la especificidad del turismo, la promoción y el marketing, la investigación, la financiación de programas, la diversificación de la oferta, el desarrollo de actividades complementarias, la formación y mentalización social, la conservación y recuperación del patrimonio y la puesta en valor de recursos (Mowforth et al., 2015; Sharpley \& Telfer, 2015; Vanhove, 2017).

En consecuencia, es sustancial cuidar el capital físico del sector y, en particular, las atracciones turísticas que generalmente son bienes públicos y 
son la base del turismo (Álvarez-Díaz et al., 2016; Belletti et al., 2017; Blasco, 2006; Giaoutzi \& Nijkamp, 2006).

\section{Referencias}

Alcaldía de Medellín, \& Centro de Ciencia y Tecnología de Antioquia (CTA). (2015, diciembre). Política Pública de Turismo Medellín 2015. https://tinyurl.com/y2oxn7kc

Álvarez-Díaz, M., González-Gómez, M., \& Otero-Giráldez, M. S. (2016). Valoración de los bienes públicos en los destinos turísticos: el excedente del consumidor de turistas europeos a Portugal. Revista Turismo $\mathcal{E}$ Desenvolvimento, (25), 77-88. https://dialnet.unirioja.es/servlet/ articulo?codigo $=6771122$

Balaguer, J., \& Cantavella-Jordá, M. (2002). Tourism as a long-run economic growth factor: the Spanish case. Applied Economics, 34(7), 877-884. https:// doi.org/10.1080/00036840110058923

Banco de la República de Colombia. (2009, marzo). Informe de la Junta Directiva al Congreso de la República. https://www.banrep.gov.co/sites/default/ files/publicaciones/archivos/ijd_mar_2009.pdf

Belletti, G., Marescotti, A., \& Touzard, J. M. (2017). Geographical indications, public goods, and sustainable development: the roles of actors' strategies and public policies. World Development, 98, 45-57. https://doi. org/10.1016/j.worlddev.2015.05.004

Bendodo Benasayag, E., Fernández Gámez, M. Á., \& Sánchez Serrano, J. R. (2019). Un análisis de la eficiencia de municipios turísticos españoles. Cuadernos de Turismo, (43), 147-168. https://doi.org/10.6018/turismo.43.06

Blasco, E. F. (2006). Turismo, bienes públicos y efectos externos de mercado. Papers de Turisme, (21), 28-61. http://www.papersdeturisme.gva.es/ojs/ index.php/Papers/article/view/321

Brida, J. G., Cortes-Jimenez, I., \& Pulina, M. (2016). Has the tourism-led growth hypothesis been validated? A literature review. Current Issues in Tourism, 19(5), 394-430. https://doi.org/10.1080/13683500.2013.868414

Brida, J. G., Monterubbianesi, P. D., \& Zapata Aguirre, S. (2011). Impactos del turismo sobre el crecimiento económico y el desarrollo. El caso de los principales destinos turísticos de Colombia. Revista de Turismo y Patrimonio Cultural, 9(2), 291-303. http://www.pasosonline.org/Publicados/9211/PASOS24.pdf\#page $=75$

Brida, J. G., Monterubbianesi, P. D., Zapata Aguirre, S., \& Giraldo Velásquez, C. M. (2010). Turismo y crecimiento económico: el caso de Antioquia. 
Turismo y Desarrollo Local, 3(7), 15. https://www.eumed.net/rev/ turydes/07/bmav.htm

Brida, J. G., Pereyra, J. S., Punzo, L. F., \& Such-Devesa, M. J. (2010). The economic contribution of tourism sector. International Journal of Hospitality and Tourism Systems, 3(1), 13-22. https://ssrn.com/abstract=1099042

Brida, J. G., Pereyra, J. S., Such Devesa, M. J., \& Zapata Aguirre, S. (2008). La contribución del turismo al crecimiento económico. Cuadernos de Turismo, (22), 35-46. https://revistas.um.es/turismo/article/view/47931

Brida, J. G., Pereyra, J. S., \& Such, M. J. (2007). Evaluating the contribution of tourism on economic growth. Anatolia: An International Journal of Tourism and Hospitality Research, 19(2), 351-356. https://doi.org/10.1080/13 032917.2008.9687079

Brida, J. G., Rodríguez-Brindis, M., Mejía-Alzate, M. L., \& Zapata-Aguirre, S. (2017). La contribución directa del turismo al crecimiento económico de Colombia: Análisis por ramas características del sector utilizando la Cuenta Satélite de Turismo-cst. Revista de Estudios Regionales, (109), 121-138. https://www.redalyc.org/pdf/755/75552738005.pdf

Capó Parrilla, J., Riera Font, A., \& Rosselló Nadal, J. (2007). Tourism and longterm growth a Spanish perspective. Annals of Tourism Research, 34(3), 709-726. https://doi.org/10.1016/j.annals.2007.02.003

Castro-Nuño, M., Molina-Toucedo, J. A., \& Pablo-Romero, M. P. (2013). Tourism and GDP: A Meta-analysis of panel data studies. Journal of Travel Research, 52(6), 745-758. https://doi.org/10.1177/0047287513478500

Cisneros-Martínez, J. D., \& Fernández-Morales, A. (2016). Concentración estacional de la demanda hotelera en Argentina. Revista de Estudios Regionales, (106), 197-221. https://www.redalyc.org/pdf/755/75547651008.pdf

Cuadrado-Roura, J. R., \& López-Morales, J. M. (2011). El turismo: un sector clave en la economía española. Papeles de Economía Española, 128, 2-20. https://dialnet.unirioja.es/servlet/articulo?codigo=3691709

De La O Barroso González, M., \& Flores Ruiz, D. (2006). La competitividad internacional de los destinos turísticos: del enfoque macroeconómico al enfoque estratégico. Cuadernos de Turismo, (17), 7-24. https://revistas. um.es/turismo/article/view/18311

Pablo-Romero, M., \& Molina, J. A. (2013, octubre 1). Tourism and economic growth: A review of empirical literature. Tourism Management Perspectives, 8, 28-41. https://doi.org/10.1016/j.tmp.2013.05.006

Díaz Olariaga, O. (2015). Análisis de la aplicación de políticas públicas en el sector turismo. El caso de Colombia. Gestión y Análisis de Políticas Públicas, (14), 17. http://www.redalyc.org/articulo.oa?id=281543007008 
Dritsakis, N. (2004). Tourism as a long-run economic growth factor: An empirical investigation for Greece using causality analysis. Tourism Economics, 10(3), 305-316. https://doi.org/10.5367/0000000041895094

Durbarry, R. (2004). Tourism and economic growth: the case of Mauritius. Tourism Economics, 10(4), 389-401. https://doi.org/10.5367/0000000042430962

Dwyer, L., Forsyth, P., \& Spurr, R. (2004). Evaluating tourism's economic effects: New and old approaches. Tourism Management, 25(3), 307-317. https://doi.org/10.1016/S0261-5177(03)00131-6

Fenalco Antioquia Operador del situr. (2019). SITUR, Sistema de Indicadores Turísticos Medellín-Antioquia. http://situr.gov.co/estadisticas/ statistics/\#!tabset_0 $=5$

Giaoutzi, M., \& Nijkamp, P. (2006). Tourism and regional development: new pathways. Routledge.

Ishikawa, N., \& Fukushige, M. (2007). Impacts of tourism and fiscal expenditure to remote islands: The case of the Amami islands in Japan. Applied Economics Letters, 14(9), 661-666. https://doi.org/10.1080/13504850500461423

Ivanov, S., \& Webster, C. (2007). Measuring the impact of tourism on economic growth. Tourism Economics, 13(3), 379-388. https://doi. org $/ 10.5367 / 000000007781497773$

Ivanov, S., \& Webster, C. (2010). Decomposition of economic growth in Bulgaria by industry. Journal of Economic Studies, 37(2), 219-227. https://doi. org $/ 10.1108 / 01443581011043609$

Ivanov, S., \& Webster, C. (2013a). Tourism's contribution to economic growth: a global analysis for the first decade of the millennium. Tourism Economics, 19(3), 477-508. https://doi.org/10.5367/te.2013.0211

Ivanov, S., \& Webster, C. (2013b). Tourism's impact on growth: The role of Globalisation. Annals of Tourism Research, 41, 231-236. https://doi. org/10.1016/j.annals.2012.12.008

Kim, H. J., Chen, M. H., \& Jang, S. C. S. (2006). Tourism expansion and economic development: The case of Taiwan. Tourism Management, 27(5), 925-933. https://doi.org/10.1016/j.tourman.2005.05.011

Llorca Rodríguez, C. M., Casas Jurado, A. C., \& Soler Domingo, A. (2013). Tourism in the economic growth of arequipa region: Assessment and development policy implications. Regional and Sectoral Economic Studies, 13(1), 5-14. https://www.scopus.com/inward/record.uri?eid=2-s2.084881566822\&partnerID $=40 \&$ md5=1614cac96db1cdc0452fc06187cdb4c9

Mejía-Alzate, M. L. (2017). Relación entre la acción económica, la estructura social y los modos de organización del subsector de la pequeña y mediana empresa que ofrecen servicios de alojamiento. En D. Herrera González, G. Ramírez Martínez, \& J. Rosas Castro (Coords.), Propuestas 
de Investigación. Una mirada a la forma de estudiar las organizaciones (pp. 505-533). Universidad de Guanajuato; Universidad Autónoma Metropolitana; Universidad Autónoma Metropolitana y Red Mexicana de Investigadores en Estudios Organizacionales. http://remineo.org/ repositorio/libros/dcoal/wp-content/uploads/2017/08/13-PropuestasDeInvestigacion.pdf

Mejía-Alzate, M. L. (2019). Las micro, pequeñas y medianas empresas en la cadena de valor global del turismo en la ciudad de Medellín, Colombia. En El Análisis Organizacional en el Desarrollo Local . Estudios de caso del entramado institucional y organizacional de Medellín, Colombia (pp. 123153). CIDE Editorial.

Mejía-Alzate, M. L., Brida, J. G., Rodríguez-Brindis, M., Giraldo-Velásquez, C., Mateus Ávila, W., Luna, O. D., \& Naranjo, L. H. (2019). Medición del impacto económico y social del turismo en la ciudad de Medellín. SITUR. https://tinyurl.com/y3t8lz54

Meyer Krumholz, D. (2004). Economía turística en América Latina y el Caribe. Universidad Externado de Colombia.

Mowforth, M., Munt, I., \& Munt, I. (2015). Tourism and Sustainability: Development, globalisation and new tourism in the third world. Routledge. https:// doi.org/10.4324/9781315795348

Mukherjee, D., \& Dutta, N. (2018). What determines governance across nations: Do economic and social globalization play a role? Economic Modelling, 69, 103-113. https://doi.org/10.1016/j.econmod.2017.09.010

Organización Mundial del Turismo (омт). (2015). Notas metodológicas de la base de datos de estadísticas de turismo. unwTo. https://www.e-unwto. org/doi/pdf/10.18111/9789284416875

Rodríguez Brindis, M. A. (2014). La contribución del turismo al crecimiento económico de México: un análisis por ramas características del sector. Nova Scientia, 7(13), 337-351. https://doi.org/10.21640/ns.v7i13.15

Sharpley, R., \& Telfer, D. J. (2015). Tourism and development: concepts and issues. Channel View Publications.

Sistema de Indicadores Turísticos Medellín-Antioquia (SITUR). (2018). Informe Septiembre 2018. SITUR.

Such-Devesa, M. J., Zapata-Aguirre, S., Risso, W. A., Brida, J. G., \& Pereyra, J. S. (2009). Turismo y crecimiento económico: un análisis empírico de Colombia. Estudios y perspectivas en turismo, 18(1), 21-35. https://papers. ssrn.com/sol3/papers.cfm?abstract_id=1092407

Uribe, S., \& Gaitán Guerrero, L. A. (2013). Impacto de la inversión extranjera directa en el sector hotelero colombiano. Revista AD-Minister, (22), 83-94. https://papers.ssrn.com/sol3/papers.cfm?abstract_id=2515724 
Vanhove, N. (2017). The economics of tourism destinations: theory and practice (Third edition). Routledge.

Webster, C., \& Ivanov, S. (2014). Transforming competitiveness into economic benefits: Does tourism stimulate economic growth in more competitive destinations? Tourism Management, 40, 137-140. https://doi.org/10.1016/j. tourman.2013.06.003

Zapata-Aguirre, S., López-Zapata, L., \& Mejía-Alzate, M. L. (2020). Tourism development in colombia: between conflict and peace. En C. Monterrubio, K. Andriotis, \& D. Stylidis (Eds.), Tourism Planning and Development in Latin America (pp. 49-65). САВ International. 